\title{
Herlyn-Werner-Wunderlich Syndrome /OHVIRA Syndrome; A Rare Urogenital Anomaly with Unusual Presentation in Two Cases with Review of Literature
}

Kamal Singh*, Sita Thakur, Anjali Soni and Ashok Verma

Department of Obstetrics and Gynaecology, Dr Rajender Prasad Govt Medical College Kangra at Tanda, Himachal Pradesh, India

*Corresponding author: Kamal Singh, Department of Obstetrics and Gynaecology, Dr Rajender Prasad Govt Medical College Kangra at Tanda, Himachal Pradesh, India, Tel: +919418033627; E-mail: singh.kamal396@gmail.com

Received date: January 18, 2016; Accepted date: January 28, 2016; Published date: February 27, 2016

Copyright: @ 2016 Singh, et al. This is an open-access article distributed under the terms of the Creative Commons Attribution License, which permits unrestricted use, distribution, and reproduction in any medium, provided the original author and source are credited.

\begin{abstract}
The OHVIRA syndrome classically occurs in the setting of uterine didelphys or more rarely, a septate uterus with incidence in various case series is $0.1-3.8 \%$. Renal agenesis is the most commonly reported urologic anomaly. The typical patient with this rare condition usually presents after menarche with nonspecific symptoms of recurrent pelvic pain or dysmenorrhea but both of our patients had atypical presentation. Sonography is frequently the initial imaging modality for evaluation of suspected Müllerian duct anomalies, MR imaging is an excellent modality for evaluating the frequently complex Müllerian duct anomalies. Treatment invariably requires surgical intervention in the form of excision of vaginal septum to relieve obstruction. In addition to relief of pain due to obstruction, surgery also reduces chances of pelvic endometriosis due to retrograde menstrual seeding. We are reporting these cases being their atypical presentation in adulthood life.
\end{abstract}

Keywords: OHVIRA syndrome; Uterine didelphys; Renal agenesis and Müllerian duct anomalies

\section{Introduction}

The OHVIRA syndrome typically represented by uterine didelphys or, more rarely, a septate uterus and is included in class III of American Fertility Society (AFS) classification. The reported incidence in various case series is $0.1-3.8 \%$ [1]. Renal agenesis is the most commonly reported urologic anomaly in OHVIRA syndrome, although other malformations, including renal duplication and multicystic dysplastic kidney, have also been described [4,5]. This syndrome is an uncommon combined Mullerian and mesonephric duct anomaly, and is usually diagnosed at or after puberty with cases presenting as abdominal pain or dysmenorrhea. These complex uterine anomalies still defied the conventional theory of urogenital development until recently when Acien theory of development of entire vagina from mesonephric ducts was postulated. The diagnostic modalities which are commonly used include ultrasonography and MRI. USG can diagnose the collection inside uterus or vagina but cannot identify the type of mullerian anomaly while MRI has multiplaner and tissue characterization ability giving precise information. Treatment invariably requires surgical intervention in the form of excision of vaginal septum to relieve obstruction. In addition to relief of pain due to obstruction, surgery also reduces chances of pelvic endometriosis due to retrograde menstrual seeding. Patients are able to have normal sexual life. Some are even able to conceive and carry pregnancy to term. Our patients had following sets of anomalies:

In case I: There is obstructed Rt hemivagina with septate uterus with ipsilateral renal agenesis.

In case II: There is obstructed Lt hemivagina with uterus didelphys with ipsilateral renal agenesis.

\section{Case I}

A 22 years nulligravida married for 9 months admitted in emergency with acute abdomen. Pain was acute in onset, moderate in intensity, continuous without any relieving or aggravating factors mainly localized in the lower abdomen and associated with vomiting. Her previous cycle was 3-4/28-30 days with dysmenorrhea and had her cycle two week back.

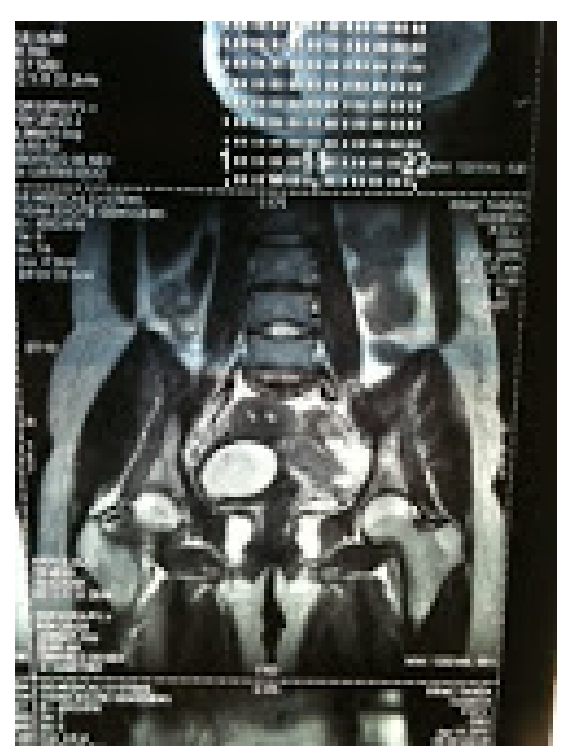

Figure 1: MR imaging of case I showing two uterine cavities with haematocolpus.

On examination, she was stable and there was no pallor. Her pulse was 84 bpm, BP- 110/80 mmhg, RR- 20/min. On examination, slight 
Citation: $\quad$ Singh K, Thakur S, Soni A, Verma A (2016) Herlyn-Werner-Wunderlich Syndrome /OHVIRA Syndrome; A Rare Urogenital Anomaly with Unusual Presentation in Two Cases with Review of Literature. Clinics Mother Child Health 13: 222. doi: $10.4172 / 2090-7214.1000222$

Page 2 of 4

tenderness with ill-defined mass felt in Rt iliac fossa with rebound tenderness plus. On speculum examination, cervix was flushed with vagina, bulge is seen right vaginal wall. On bimanual examination cystic non tender mass felt in Rt fornix with restricted mobility projecting in vagina. So possibility of appendicular lump and Rt TO mass kept. Her investigation was normal. UPT was negative.

USG shows E/O acute appendicitis with non-visualization of Rt kidney, with cystic mass showing internal echoes on the Rt side of pelvis and a bicorn ate uterus with blood in the cavity. On MRI finding were s/o complete septate uterus with haematocolpus of Rt hemivagina with absent Rt kidney and solitary Lt kidney showing hypertrophy i.e., OHVIRA syndrome (Figure 1).

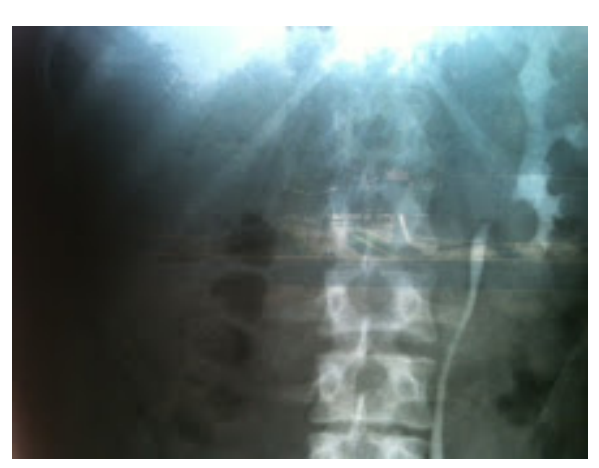

Figure 2: IVP of case I showing Lt renal collecting system with ureter.

On IVP non visualization of Rt kidney and Rt ureter likely Rt renal agenesis (Figure 2). She was treated conservatively for acute appendicitis and after 6 weeks subjected for diagnostic laparoscopy (Figure 3) and drainage of haematocolpus.

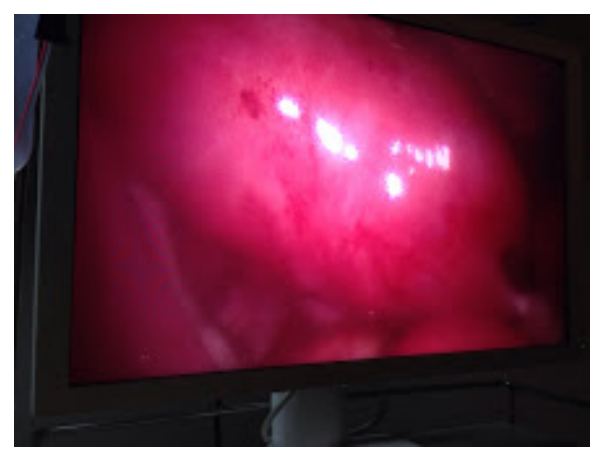

Figure 3: Laparoscopy of case I showing single uterus.

On laparoscopy there was single uterus with normal bilateral fallopian tubes and ovaries. Haematocolpus which was around $6 \times 6$ $\mathrm{cm}$ on Rt side drained after giving cruciate incision, around $90-100 \mathrm{ml}$ of dark coloured blood drained. Foley's cathter kept for 2 weeks. After 2 weeks, catheter removed and excision of septum was done in $2^{\text {nd }}$ sitting. On follow up she had normal cycle without any evidence of haematocolpus.

\section{Case II}

A 21 years G2P0010 at POG 8 weeks with previous Ist trimester abortion admitted in emergency with acute abdomen. She was married for one year and had her normal previous cycle. She had amenorrhea for two month and UPT was positive. Her GPE were unremarkable.

On per abdominal examination shows mild tenderness lower abdomen without any rigidity or muscle guarding. On $\mathrm{P} / \mathrm{V}$ examination there was ill defined tender, cystic mass felt in left fornix with restricted mobility without cervical motion tenderness. So possibility of ectopic pregnancy kept and further investigation advised.

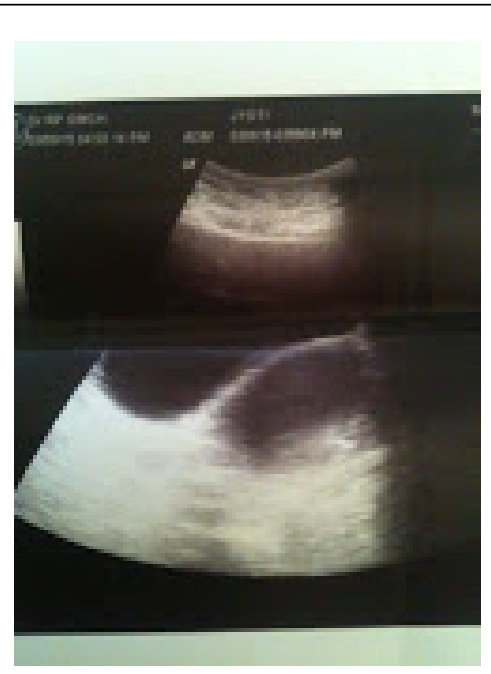

Figure 4: USG of case II showing two uteri.

On USG there were e/o two uterine cavities without any gestational sac (Figure 4), with left haematocolpus (Figure 5) with left renal agenesis suggestive of OHVIRA syndrome. Her MRI confirms the diagnosis. She underwent laparoscopy proceeded by drainage of haematocolpus in two step protocol. Her postoperative period was uneventful. On further follow up she had normal menstrual cycle and on examination no evidence of haematocolpus.

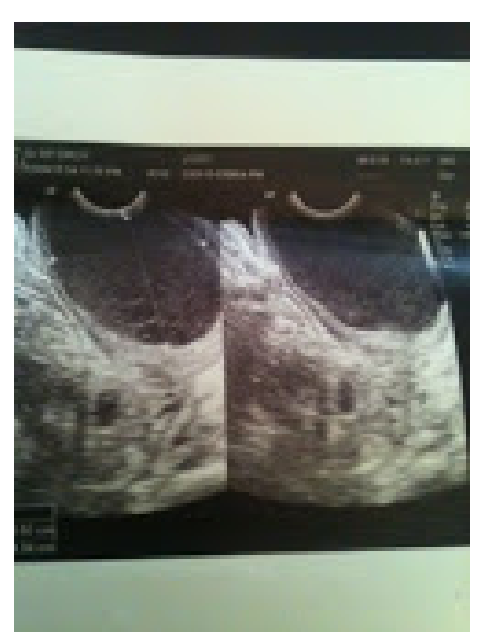

Figure 5: USG of case II showing haematocolpus. 
Citation: $\quad$ Singh K, Thakur S, Soni A, Verma A (2016) Herlyn-Werner-Wunderlich Syndrome /OHVIRA Syndrome; A Rare Urogenital Anomaly with Unusual Presentation in Two Cases with Review of Literature. Clinics Mother Child Health 13: 222. doi: $10.4172 / 2090-7214.1000222$

Page 3 of 4

\section{Discussion}

The syndrome of obstructed hemivagina and ipsilateral renal anomaly was initially reported in 1922 and is known as HerlynWerner-Wunderlich syndrome or, more recently, by the acronym OHVIRA. The reported incidence in various case series is $0.1-3.8 \%$ [1]. The OHVIRA syndrome classically occurs in the setting of uterine didelphys or, more rarely, a septate uterus [2]. Renal agenesis is the most commonly reported urologic anomaly in OHVIRA syndrome, although other malformations, including renal duplication and multicystic dysplastic kidney, have also been described [4,5]. In a reported series of 27 cases, the age at diagnosis varied from 10 to 29 years, with a median age of 14 years [5]. The typical patient with this rare condition usually presents after menarche with nonspecific symptoms of recurrent pelvic pain or dysmenorrhea from progressive distension of the obstructed hemivagina. Early and accurate diagnosis is important because prompt therapeutic intervention can relieve symptoms, prevent further complications related to chronic obstructed hematocolpos (such as endometriosis and pelvic adhesions), and maintain reproductive capacity. Delays in diagnosis were blamed on lack of knowledge of the disease, regular menstruations in context of an incomplete vaginal outlet obstruction, and slow extension of hematocolpos.

By the end of the $20^{\text {th }}$ century, embryology of female urogenital system was accepted to be a truly-settled issue. While the uterocervical development was believed to be from paired paramesonephric ducts; sinovaginal bulb from urogenital sinus was thought to develop into lower vagina; the kidneys and ureters were believed to arise from wolffian $\mathrm{pro} / \mathrm{meso} / \mathrm{metanephros}$. Aberration in vertical or horizontal fusion or arrest of paramesonephric ducts during course of development was understood to successfully explain various uterine anomalies. Some complex uterine anomalies such as OHVIRA syndrome (Obstructed Hemi Vagina with Ipsilateral Renal Agenesis) still defied the conventional theory of urogenital development until recently when Acien theory of development of entire vagina from mesonephric ducts was postulated [3]. Vaginal embryonic development has become a topic of hot debate consequent to propounding of new theory of vaginal origin by Acien (Figure 6) [6].

The classic theory was found inadequate in explaining complex mullerian malformations like OHVIRA and their coexistence with renal anomalies. Acien proposed that while uterus and cervix were derived from fused-paired paramesonephric ducts $\left(2^{\text {nd }}\right.$ part) and divergent distal paramesonephric ducts ( $3^{\text {rd }}$ part), the vagina was completely of mesonephric (wolffian) origin although its lining did reveal mullerian cells derived from mullerian tubercle. Hence, paramesonephric ducts do not contribute to formation of the vagina although mullerian tubercle does, such that the vagina is lined by cells from mulleria tubercle. This theory has been further reinforced by data published by Sánchez in his experiments with female rats using histoimmunochemistry techniques. An early failure of metanephric diverticulum to develop (around 5 weeks) from mesonephric duct results in agenesis of ureteric bud, which leads to agenesis of ipsilateral ureter and kidney. Sonography is frequently the initial imaging modality for evaluation of suspected Müllerian duct anomalies. It can be difficult to differentiate a hematometra with thin, stretched myometrium from hemorrhagic adnexal masses on ultrasound. So MR imaging is an excellent modality for evaluating the frequently complex Müllerian duct anomalies, given its multi planar capability, superior tissue characterization, and large field of view.

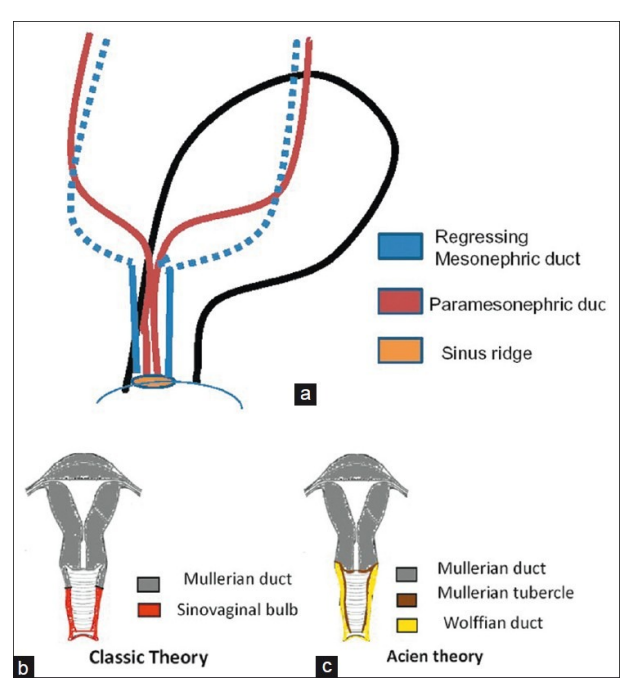

Figure 6: (a) Embryological development of the female urogenital system (b) Classic theory of development of uterus and vagina (c) Acien theory of development of uterus and vagina.

MR imaging can accurately depict the uterine contour, shape of uterine cavity, and associated cervical and vaginal anomalies, important in the classification of Müllerian duct anomalies and in surgical planning. In addition, MR imaging can provide tissue characterization of the septa, characterize the contents of obstructed cavities (for example, simple fluid versus blood), and easily detect any coexisting renal and/or urethral abnormalities. Primary management of the OHVIRA syndrome is requires surgical intervention in the form of excision of vaginal septum to relieve obstruction. Historically, surgeons advocated a two-stage procedure, with the initial surgery to reduce the hematocolpos and the second to re-sect the excess septum after a period of wound-healing and vaginal remodelling.

A single-stage vaginoplasty, involving complete resection of the septum in a single procedure can be done. In both of our patients we follow the two step protocol. In addition to relief of pain due to obstruction, surgery also reduces chances of pelvic endometriosis due to retrograde menstrual seeding. Patients are able to have normal sexual life. In fact, hemihystrectomy, done in earlier times, is no more preferred now as the reported incidence of pregnancy in both horns is almost equal. Altchek and Paciuc have reported pregnancy occurring twice in a previously-obstructed didelphys uterus after surgical correction. The surgeon must, therefore, make every effort to preserve the obstructed uterus.

\section{Conclusion}

In conclusion, OHVIRA syndrome is a rare congenital urogenital anomaly with wide range of clinical presentation but simple surgical management. Imaging, particularly an MRI plays a major role in diagnosis, which is often missed clinically. The complex mullerian anomalies have been difficult to diagnose correctly without help of imaging. Appropriate surgery is a single stage procedure to either excise or completely divide the obstructing septum. Early correct diagnosis is required to relieve the symptoms and prevent complications, caused by retrograde menstruation which may result in endometriosis and, also, preserve sexual and conception abilities. 
Citation: Singh K, Thakur S, Soni A, Verma A (2016) Herlyn-Werner-Wunderlich Syndrome /OHVIRA Syndrome; A Rare Urogenital Anomaly with Unusual Presentation in Two Cases with Review of Literature. Clinics Mother Child Health 13: 222. doi: $10.4172 / 2090-7214.1000222$

Page 4 of 4

\section{References}

1. Resetkova N, Christianson M, Kolp L (2012) Uterine didelphys with obstructed hemivagina and ipsilateral renal agenesis with hydronephrosis. Fertil Steril 97: 30-31.

2. Strassmann EO (1966) Fertility and unification of double uterus. Fertil Steril 17: 165-176.

3. Hoeffel C, Olivier M, Scheffler C, Chelle C, Hoeffel JC (1997) Uterus didelphys, obstructed hemivagina and ipsilateral renal agenesis. European Journal of Radiology 25: 246-248.
4. Li S, Qayyum A, Coakley FV, Hricak H (2000) Association of renal agenesis and mullerian duct anomalies. J Comput Assist Tomogr 24: 829-834.

5. Vercellini P, Daguati R, Somigliana E, Viganò P, Lanzani A, et al. (2007) Asymmetric lateral distribution of obstructed hemivagina and renal agenesis in women with uterus didelphys: institutional case series and a systematic literature review. Fertility Sterility 87: 719-724.

6. Acién P (1992) Embryological observations on the female genital tract. Hum Reprod 7: 437-445. 